\title{
Prenatal and infant exposure to ambient pesticides and autism spectrum disorder in children: population based case-control study
}

\author{
(c) (1) ()
}

In this article by von Ehrenstein and colleagues (BMJ 2019;364, doi:10.1136/bmj.1962, published 20 March and in the 23 March print issue), an oversight by coauthor Beate Ritz led to the omission of a potentially perceived conflict of interest on the declaration form (relating to other relationships or activities). The competing interest statement has since been updated in the online article and PDF. 